\title{
Recent results from GERDA Phase II
}

\section{Christoph Wiesinger* for the GERDA collaboration}

Physik Department and Excellence Cluster Universe, Technische Universität München, D-85748

Munich, Germany.

E-mail: christoph.wiesingeretum.de

\begin{abstract}
The combination of an ultra-low background environment with active background suppression techniques allows GERDA (GERmanium Detector Array) to perform a background-free search for neutrinoless double beta decay of ${ }^{76} \mathrm{Ge}$. GERDA is located at the Laboratori Nazionali del Gran Sasso (LNGS) of INFN in Italy. In Phase II $35.6 \mathrm{~kg}$ of isotopically enriched high purity germanium detectors are operated in a liquid argon bath. The background index in the regionof-interest is $<10^{-3}$ counts/(keV· $\left.\mathrm{kg} \cdot \mathrm{yr}\right)$. No signal has been found in the data corresponding to $58.9 \mathrm{~kg} \cdot \mathrm{yr}$ of exposure and in combination with data from Phase I a new lower limit on the half-life of neutrinoless double beta decay in ${ }^{76} \mathrm{Ge}$ of $\mathrm{T}_{1 / 2}>0.9 \cdot 10^{26} \mathrm{yr}(90 \%$ C.L.) has been established. GERDA is the first neutrinoless double beta decay experiment that surpassed a half-life sensitivity of $>10^{26} \mathrm{yr}$.
\end{abstract}

Neutrino Oscillation Workshop (NOW2018)

9 - 16 September, 2018

Rosa Marina (Ostuni, Brindisi, Italy)

${ }^{*}$ Speaker. 


\section{Neutrinoless double beta decay}

In double beta $(\beta \beta)$ decay a nucleus ${ }_{Z}^{A} \mathrm{X}$ decays into its daughter nucleus ${ }_{Z+2}^{A} \mathrm{Y}$. In the standard model this occurs with the emission of two electrons and two anti-neutrinos. An observation of a neutrinoless mode, so-called neutrinoless double beta $(0 v \beta \beta)$ decay, would imply a violation of lepton number conservation and establish the Majorana nature of neutrinos [1]. In this case the two electrons would share the full Q-value $\left(\mathrm{Q}_{\beta \beta}\right)$ of the decay.

\section{Experimental approach}

Isotopically modified High-Purity Germanium (HPGe) detectors offer unique properties to search for $0 v \beta \beta$ decay. Enrichment in the $\beta \beta$ isotope ${ }^{76} \mathrm{Ge}$ is well established and detectors that simultaneously constitute the source and the absorber yield a very high detection efficiency. The energy resolution is of $\mathscr{O}(0.1 \%)$ for the $0 v \beta \beta$ decay signal at $Q_{\beta \beta}=2039 \mathrm{keV}$.

a)

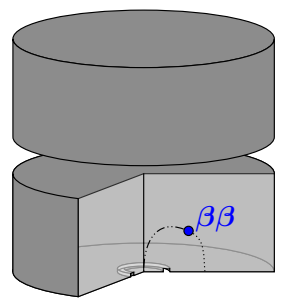

b)

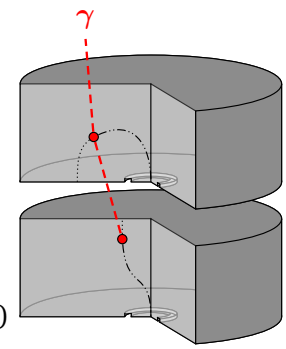

c)

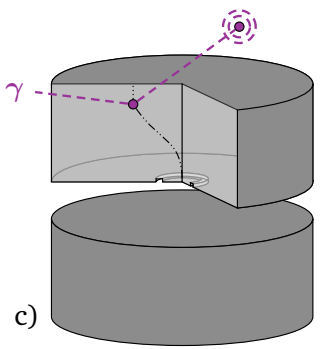

d)

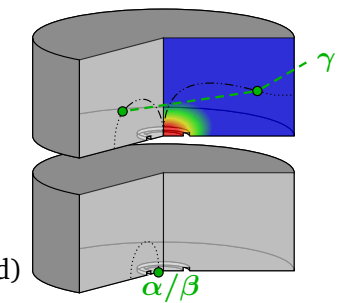

Figure 1: Event topologies and active background suppression in GeRDA Phase II. Double beta $(\beta \beta)$ decay events in the germanium bulk generate a very localized energy deposition in one HPGe detector (a). Coincident energy depositions in several HPGe detectors, e.g. Compton scattered $\gamma$ 's, are rejected in terms of detector anti-coincidence (b). The possibility to detect a coincident energy release in the detector surroundings, e.g. by liquid argon (LAr) scintillation, enables to suppress events with partial energy deposition in germanium (c). The internal field configuration of the HPGe detectors enables to discriminate events with multiple energy depositions in one crystal as well as surface events by pulse shape discrimination (PSD) (d). The drift path of charge carriers in the HPGe detectors is indicated.

Although HPGe ionization detectors do not allow particle identification, background can be discriminated by its event topology (see Fig. 1). The large stopping power of germanium results in very localized energy depositions of $\mathscr{O}\left(1 \mathrm{~mm}^{3}\right)$ from electrons released in $\beta \beta$ decay. The mean free path of $\gamma$ radiation at $\mathrm{MeV}$-energies is generally much larger and results in a more widespread event topology. The granularity of several detectors in an array configuration allows to reject events that deposit energy in more than one HPGe detector. The capability for anti-coincidence rejection is largely enhanced by embedding the HPGe crystals in an active medium itself. In GERDA the HPGe detectors are operated in liquid argon (LAr), an excellent scintillator. Additionally, the pulse shape of HPGe signals carries information on the charge carrier drift path and consequently on the interaction point within the detector. This allows to identify and suppress events arising from multiple energy depositions within the same crystal as well as the rejection of surface $\alpha$ and $\beta$ events by pulse shape discrimination (PSD) [2]. 


\section{The GERDA setup}

GERDA is located in Hall A of the LNGS underground laboratoy of INFN in Italy. LNGS provides a rock overburden of $3500 \mathrm{~m}$ water equivalent. Additional shielding is provided by a $590 \mathrm{~m}^{3}$ water tank. It efficiently absorbs neutrons and is instrumented with photomultipliers (PMTs) to serve as Cherenkov veto for cosmic muons. Inside the water tank a $64 \mathrm{~m}^{3}$ cryostat filled with LAr is situated. The germanium detector array is inserted into the cryostat via an air-tight lock from a clean room on top of the experiment. The LAr provides another layer of high-purity shielding and at the same time acts as coolant for the HPGe detectors. In Phase II the array comprises of 40 HPGe detectors in a seven string configuration, from which 37 detectors with a total mass of $35.6 \mathrm{~kg}$ are made from enriched germanium with $\sim 87 \%{ }^{76} \mathrm{Ge}$. They can be divided in 7 (semi-)coaxial detectors from former experiments and 30 custom-made Broad Energy germanium (BEGe) detectors. BEGe detectors posses superior performance in terms of energy resolution and PSD, but have lower detector mass. All materials close to the array are reduced to a minimum and selected for highest radiopurity. Together with the array a liquid argon light instrumentation has been inserted into the cryostat. Scintillation light emerging from the array surroundings is detected with PMTs and wavelength shifting fibers coupled to silicon photomultipliers [3].

\section{Performance and results}

The data taking of GERDA Phase II started in December 2015. Until April 2018, $58.9 \mathrm{~kg} \cdot \mathrm{yr}$ of analysis data were collected. Events in an energy interval $\pm 25 \mathrm{keV}$ around $\mathrm{Q}_{\beta \beta}$ are stripped from the data stream and only analyzed after all input parameters are frozen. The classification of events from previously unblinded data [4] has not been changed. The main datasets contain an exposure of $23.1 \mathrm{~kg} \cdot \mathrm{yr}$ from coaxial and $30.8 \mathrm{~kg} \cdot \mathrm{yr}$ from BEGe type detectors. Weekly calibrations with ${ }^{228} \mathrm{Th}$ sources guarantee a properly defined energy scale over the full data taking period. Between calibrations, instabilities are tracked by test pulses injected into the front end electronics. Instable detectors and periods are removed from the analysis. The energy reconstruction is performed with a digital filter optimized for low-frequency noise rejection [5]. The full-width at half-maximum (FWHM) at $\mathrm{Q}_{\beta \beta}$ is 3.6(1) keV for coaxial and 3.0(1) keV for BEGe detectors (see Fig. 2).

Events with a registered LAr light emission that is above a threshold individually set for each light detector are rejected. Random coincidence rejection due to naturally present ${ }^{39} \mathrm{Ar}$ and dark rate is $2.3(1) \%$. Pulse shape discrimination for BEGe detectors is carried out by a mono-parametric cut based on the ratio between current pulse amplitude and total collected energy. The cut values are determined from calibration data to accept $90 \%$ of the double escape peak of ${ }^{208} \mathrm{Tl}$ - a proxy for signal-like single-site events. Rejection of $\alpha$ surface events is achieved by cutting events $>4 \sigma$ from the single-site distribution. For coaxial detectors an artificial neural network analysis trained on calibration data is used. It is completed by a rise time cut for surface $\alpha$ rejection. The acceptance for signal events is $71(4) \%$ for coaxial and $88(3) \%$ for BEGe detectors. The background level is determined in an energy window from 1930 to $2190 \mathrm{keV}$ excluding $\pm 5 \mathrm{keV}$ around $\mathrm{Q}_{\beta \beta}$ and the known $\gamma$ lines from ${ }^{208} \mathrm{Tl}$ and ${ }^{214} \mathrm{Bi}$ at $2104 \mathrm{keV}$ and $2119 \mathrm{keV}$ respectively. The background index after active background rejection cuts is $5.7_{-2.6}^{+4.1} \cdot 10^{-4}$ counts $/(\mathrm{keV} \cdot \mathrm{kg} \cdot \mathrm{yr})$ for coaxial and $5.6_{-2.4}^{+3.4} \cdot 10^{-4}$ counts/(keV·kg $\cdot \mathrm{yr}$ ) for BEGe detectors (see Fig. 3). A combined unbinned maximum 


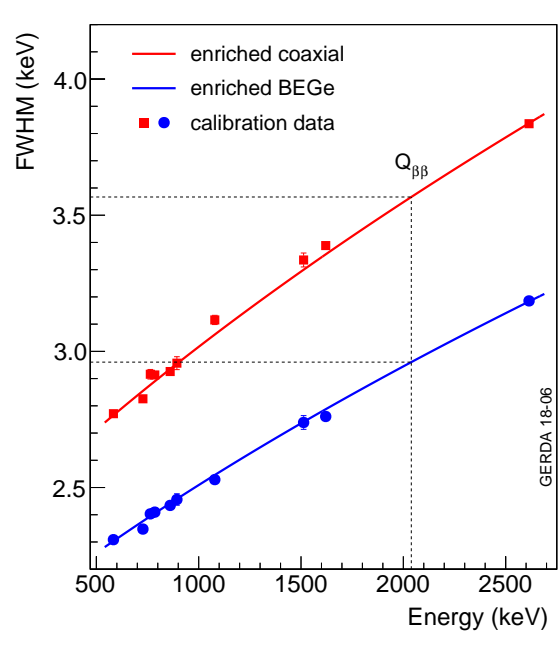

Figure 2: Resolution curves for GERDA Phase II detector types. The values are obtained from weekly calibrations with ${ }^{228} \mathrm{Th}$ sources. BEGe detectors feature superior resolution.

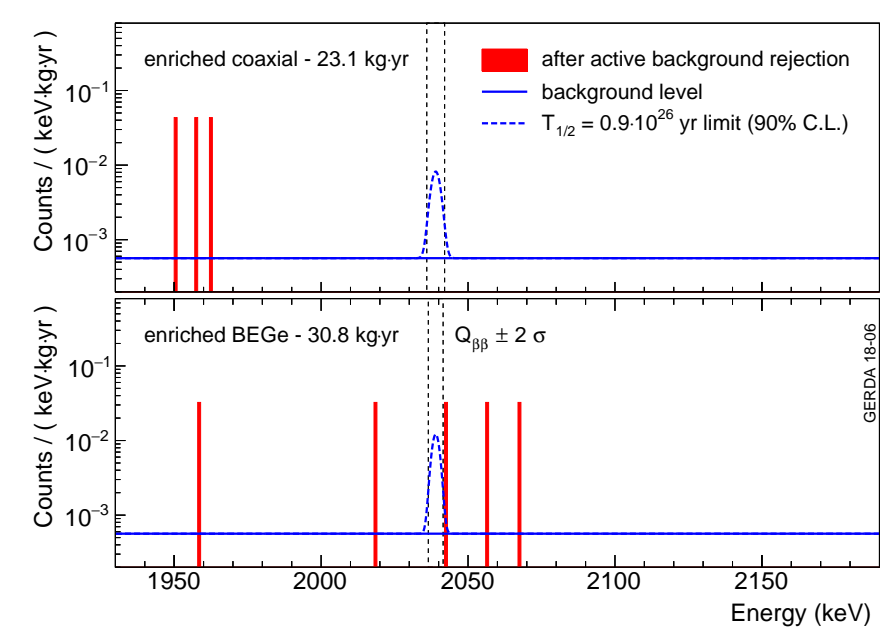

Figure 3: Energy spectra around $\mathrm{Q}_{\beta \beta}$ for the main GERDA Phase II datasets. The background level and a hypothetical signal corresponding to the lower half-life limit from the combined fit to all GERDA data is indicated. The closest event is more than $2 \sigma$ from $\mathrm{Q}_{\beta \beta}$.

likelihood fit using a Gaussian signal on a flat background was performed on the full GERDA data of $82.4 \mathrm{~kg} \cdot \mathrm{yr}$. The signal efficiency including active volume, enrichment and containment fraction as well as the above mentioned signal acceptances is $48(4) \%$ for coaxial and $60(2) \%$ for BEGe detectors. The frequentist analysis yields a lower limit on the half-life of $0 v \beta \beta$ decay of ${ }^{76} \mathrm{Ge}$ of $\mathrm{T}_{1 / 2}>0.9 \cdot 10^{26} \mathrm{yr}(90 \%$ C.L. $)$, which is below the median sensitivity of $1.1 \cdot 10^{26} \mathrm{yr}$.

\section{Conclusions and outlook}

The approach of combining elaborate passive shielding with active background suppression enabled GERDA to reduce the background to an unprecedented level of $<10^{-3}$ counts/(keV $\left.\cdot \mathrm{kg} \cdot \mathrm{yr}\right)$. With no signal found, a new limit on the half-life of $0 v \beta \beta$ decay of ${ }^{76} \mathrm{Ge}$ was derived. After an upgrade in spring 2018 the background-free exploration of half-lifes beyond $10^{26} \mathrm{yr}$ continues. In 2020 the experimental infrastructure will be handed over to the Large Enriched Germanium Experiment for Neutrinoless Double-Beta Decay (LEGEND), which will expand this reach [6].

\section{References}

[1] J. Schechter and J. W. F. Valle, Phys. Rev. D25, p. 2951 (1982).

[2] M. Agostini et al. (GERDA collaboration), Eur. Phys. J. C73, p. 2583 (2013).

[3] M. Agostini et al. (Gerda collaboration), Eur. Phys. J. C78, p. 388 (2018).

[4] M. Agostini et al. (GERDA collaboration), Phys. Rev. Lett. 120, p. 132503 (2018).

[5] M. Agostini et al. (GERdA collaboration), Eur. Phys. J. C75, p. 255 (2015).

[6] N. Abgrall et al. (LEGEND collaboration), AIP Conf. Proc. 1894, p. 020027 (2017). 